\author{
Pascal Frei ${ }^{\mathrm{a}}$ \\ Gerhard Rogler $^{b}$
}

\title{
Darm-Detoxifikation: Mythos oder sinnvolle Therapie?
}

chen, über «Entgiftungen», «DetoxTherapien» und «Entschlackungskuren» einen gesundheitlichen Benefit zu erreichen.

\section{Hintergrund der Behandlungsansätze}

Entgiftungsbehandlungen basieren auf der alten, schon in der Antike vorhandenen Vorstellung, dass eine Obstipation zu einem zu langen Verweilen toxischer Substanzen im Darm führe. Dies würde dann konsekutiv eine vermehrte Aufnahme toxischer Substanzen im Sinne einer "Autointoxikation» verursachen [1]. Solche Überlegungen erscheinen auf den ersten Blick vielen Menschen plausibel (obwohl für sie keine wissenschaftliche Evidenz existiert) und lassen sich gut vermarkten («clean on the inside beautiful on the outside»). Aus physiologischen Untersuchungen wissen wir allerdings, dass die wesentliche Absorptionsfunktion des Darmes nicht im Kolon, sondern im Dünndarm lokalisiert ist, welcher für Therapien wie eine Kolon-Hydro-Therapie nicht zugänglich ist. Zudem konnte bisher keine wissenschaftliche Evidenz gewonnen werden, die zeigen würde, dass eine Obstipation tatsächlich zur vermehrten Resorption bakterieller Toxine oder von Nahrungsmittelzusätzen führen könnte. Insofern muss man die Annahme, dass eine chronische Intoxikation über das Kolon vorhanden sei, sehr kritisch sehen. Eine «Detoxifikation» ergibt aber nur Sinn, wenn man von einer solchen Intoxikation ausgeht. Möglicherweise ist das Bedürfnis nach «Detoxifikation» oder «Entgiftung» eine scheinwissenschaftlich transformierte Erscheinung alter Reinigungsriten, die es in nahezu allen Kulturen gab und gibt.

Verschiedene Mechanismen werden diskutiert oder gar angepriesen, um eine «Detoxifikation» zu erreichen. Interessanterweise widersprechen sich die Therapieansätze teilweise. So sollen abführende Entgiftungsstrategien die Noxen aus dem Körper spülen, wohingegen Quellmittel eigentlich das Gegenteil bewirken (sie füllen das Kolon, statt es direkt mechanisch zu entleeren). Angepriesen wird dies als «darmreinigendes Ballaststoffergänzungsmittel».

Die Vorstellung des «Freispülens» ist offensichtlich stark vereinfacht. Der Darm hat auch die Funktion, Noxen in einem kompakten Stuhl zu bündeln. Solche Kotballen können aber durch mechanische Reize bei der transanalen Volumengabe aufgeweicht werden, was dann eine Freisetzung potenzieller Noxen und damit einen erhöhten Kontakt von Noxen mit der Darmmukosa zur Folge hat $[2,3]$.

Trotz aller angebrachten Skepsis sollte sich die Schulmedizin überlegen, ob die Häufigkeit der durchgeführten «Detox»-Therapien und ähnlicher Verfahren nur auf gutem Marketing beruht oder ob der Markt durch einen realen Nutzen oder ein reales Bedürfnis gestützt wird. Eine Umfrage unter Patienten, die eine Kolon-Hydro-Therapie machten, zeigte, dass viele von ihnen unzufrieden mit der Schulmedizin waren [4]. Es ist jedoch 
nicht adäquat, den Sinn einer Therapie über die Häufigkeit ihrer Anwendung oder die Zufriedenheit der Anwender zu rechtfertigen. Auch darf die Beobachtung, dass viele Patienten von einer Stuhlregulation profitieren oder viele Patienten sich nach dem Abführen im Hinblick auf eine Koloskopie wohlfühlen, nicht extrapoliert und geschlussfolgert werden, dass Detoxifkationen in ihrer Wirksamkeit belegt wären. Getreu einem kürzlich publizierten Editorial in dieser Zeitschrift mit dem Titel «Entwicklung von integrativer Medizin bedeutet auch Verantwortung für Qualität» [5] machen wir uns darum auf die schwierige Suche nach Evidenz. Literatur findet sich in der Datenbank PubMed vor allem mit den Termini «detoxification» und «bowel cleansing».

Die Durchführung einer «Detoxifikation» ist grundsätzlich von oral oder von rektal (auch bekannt als KolonHydro-Therapie) möglich. Nicht eingehen möchte dieser Artikel auf Ernährungsversuche zur Detoxifikation und auf Probiotika bzw. AntibiotikaTherapien. Hierfür sei auf den Beitrag von Biedermann et al. [6] als Teil des Themenschwerpunktes «Gastroenterologie» in dieser Ausgabe verwiesen.

\section{Orale Therapien}

Es wurden bisher keine Studien publiziert, die zeigen könnten, dass ein orales Reinigen («bowel cleansing») einen positiven Effekt auf die allgemeine Gesundheit hätte [7]. Hingegen gibt es interessante Untersuchungen, die belegen, dass man mit Abführen von oral nicht nur die Quantität der Darmkeime ändert, sondern auch die Qualität der Darmflora oder des DarmMikrobioms im Sinne der Keimzusammensetzung. Jalanka et al. [8] zeigten an einer Untersuchung von 23 gesunden Probanden, dass die Keimzahl durch ein orales Abführen um das 31-Fache reduziert wird. Jeder fünfte Proband verlor nach dem Abführen sein spezifisches Keimmuster in der
Darmflora. Innert 2 Wochen war das Mikrobiom aber wieder aufgebaut. Interessanterweise waren die Veränderungen grösser, wenn das Abführen an einem Tag und nicht über mehrere Tage hinweg geschah [8].

Drago et al. [9] zeigten mit einer anderen Methodik, dass ein Abführen (wie es z.B. zur Darmspiegelung notwendig ist; etwa mit 4L Moviprep) auch nach einem Monat noch einen Einfluss auf die Keimzusammensetzung haben kann. Dabei wurde nachgewiesen, dass unter anderem auch die Zahl an Lactobazillen reduziert wird [9]. Hier handelt es sich jedoch um eine Bakterienspezies, die als Probiotika in gutem Ruf steht, vielfach z.B. zum Schutz vor Antibiotika-verursachten Durchfällen eingesetzt wird und einen günstigen Effekt auf die Gesundheit haben soll. Dies zeigt wiederum, dass wir aus ärztlich-biologischer Sicht die Wirkung der oralen Darmspülung noch nicht genügend verstehen, um über diese Manipulation voraussagbar günstige Effekte zu erzielen.

Zusammenfassend zeigen diese $\mathrm{Da}-$ ten, dass das orale Abführen zur Detoxifikation wohl einen Effekt auf das Mikrobiom haben kann. Dieser Effekt ist bisher aber weder an grossen Kollektiven belegt, noch gibt es Hinweise, dass dieser Effekt über einen längeren Zeitpunkt bestehen bleibt. Dass dieser «Effekt» aber eine «Toxinreduktion» oder «Entgiftung» bewirkt und/oder nachhaltig ist, ist noch wesentlich schlechter belegt. Darüber hinaus ist nicht klar, welche denn die relevanten Toxine sein sollen, die durch die Darmspülung «ausgeschwemmt» werden sollten. Ein repetitives Abführen, wollte man einen langfristigen Effekt erzielen, ist kaum machbar bei dem meist unangenehmen Procedere.

\section{Rektale Therapien}

Rektal applizierte Therapien erreichen bei einer Zäpfchen-Therapie primär das Rektum. Es ist aber erstaunlich, dass auch kleinvolumige Schäu- me und Einläufe das Kolon häufig über die linke Flexur hinaus erreichen [10]. Dies ist unter anderem mit nuklearmedizinischen Studien von markierten Rektaltherapien belegt.

Viele Nachfragen kommen im klinischen Alltag zur sogenannten KolonHydro-Therapie, einer bewusst grossvolumigen Therapie mit Wassereinläufen. Anfragen hierzu stammen vielfach von Patienten mit chronisch-entzündlichen Darmerkrankungen (Inflammatory Bowel Disease (IBD)), vor allem aber von Patienten mit Blähungen oder Verstopfung (Reizdarmsyndrom oder Irritable Bowel Syndrome (IBS)) bzw. Divertikelleiden oder einfach von Menschen mit dem Wunsch, sich mittels solcher Detox-Kuren etwas Gutes zu tun. Diese Therapieansätze sind wie erwähnt schon sehr alt.

Publizierte Reviews sind dabei teils positiv [1], teils sehr kritisch [2].

Es gibt keine klinischen Studien, die zeigen, dass Kolon-Hydro-Therapien einen Effekt auf die allgemeine Gesundheit haben [7]. Für die chronischentzündlichen Darmerkrankungen zeigte eine 2010 publizierte Übersichtsarbeit, dass es keine Evidenz für die Wirksamkeit der Kolon-HydroTherapie gibt [11].

Zudem bestehen im Rahmen dieser Therapie grundsätzliche und signifikante Risiken. Hierzu gehören Infektionen, Elektrolytstörungen und Volumenprobleme, die durch die grossvolumigen Einläufe hervorgerufen werden können.

Eine Therapie einer Obstipation hat per se nichts mit einer Detoxifikation zu tun und kann und soll primär oral erreicht werden. Dies ist die Domäne der pflanzlichen Quellmittel wie Leinsamen, Flohsamen usw.

Eine Kolon-Hydro-Therapie der Divertikelkrankheit kann aus schulmedizinischer Sicht nicht empfohlen werden. Einerseits sollte nicht über Manipulationen eine Divertikel-Perforation riskiert werden; andererseits ist die Vorstellung, Divertikel freizuspülen, zu mechanistisch. Dies wird allein dadurch belegt, dass bei der Ko- 
loskopie (nach ergo mehreren Litern Durchfall) häufig multipelste Divertikel immer noch Stuhlreste enthalten (Abb. 1).

Zusammenfassend gibt es also für rektale Therapien wie die Kolon-Hydro-Therapie weder bei gesunden Menschen noch für Patienten mit einer spezifischen Erkrankung Evidenz für deren Wirksamkeit. Dass solche Therapien häufig «guttun», mag über den Placeboeffekt erklärbar sein, aber auch, weil viele Menschen obstipiert sind und darum die Hydrotherapie kurzfristig als angenehm erachten. Auch wenn die Risiken statistisch klein scheinen, sind sie unter Umständen für den Betroffenen gravierend. Darum wären sie aus schulmedizinischer Sicht nur gerechtfertigt, wenn der Nutzen klar belegt wäre. Es sei erwähnt, dass auch für Detoxifikationen mit anschliessenden aufwendigen probiotischen Aufbaukuren die Evidenz fehlt. Bei limitiertem Nutzen und signifikantem Risiko sind die Preise, die teilweise für eine solche Therapie gefordert (und auch bezahlt) werden, inakzeptabel.

\section{Literatur}

1 Bazzocchi G, Giuberti R: Irrigation, lavage, colonic hydrotherapy: from beauty center to clinic? Tech Coloproctol 2017;21:1-4.

2 Seow-Choen F: The physiology of colonic hydrotherapy. Colorectal Dis 2009;11:686-688.

3 Johnson DA, Barkun, AN, Cohen LB, et al.; US Multi-Society Task Force on Colorectal Cancer: Optimizing adequacy of bowel cleansing for colonoscopy: recommendations from the US multi-society task force on colorectal cancer. Gastroenterology 2014;147:903-924.

4 Taffinder NJ, Tan E, Webb IG, McDonald PJ: Retrograde commercial colonic hydrotherapy. Colorectal Dis 2004;6:258-260.

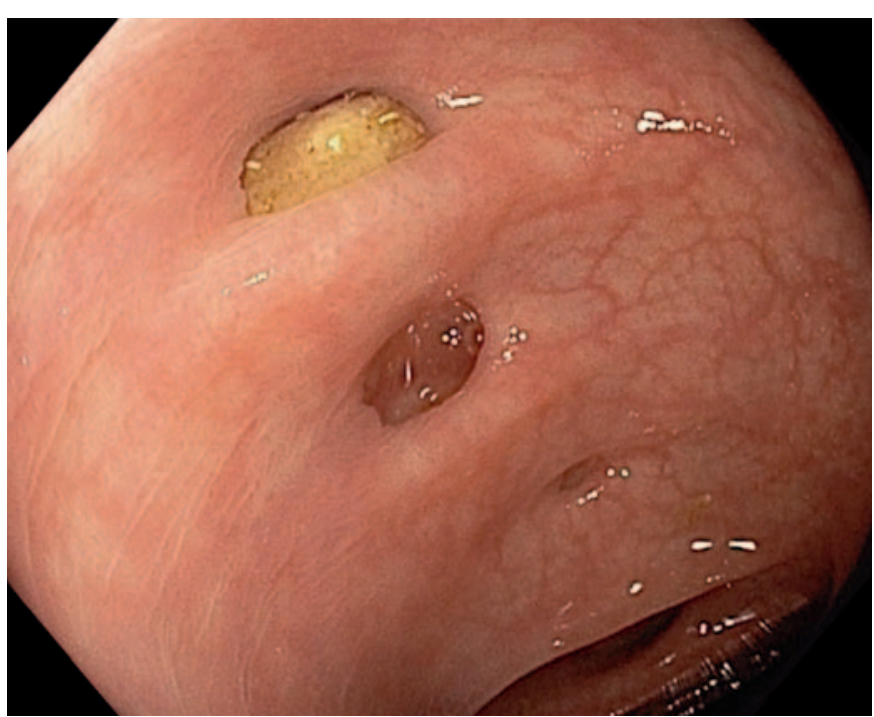

Atuhl trotz Stuhl trotz Abführen.

\section{Fazit}

Obschon Detoxifikationstherapien eine lange Tradition haben, gibt es keine wissenschaftliche Evidenz für deren klinische Wirksamkeit, insbesondere nicht bezogen auf klinisch relevante Endpunkte. Solange der klinische Nutzen nicht eindeutig belegt ist, müssen die Risiken und Kos- ten kritisch hinterfragt werden. Solange die Kosten vom Individuum und nicht von der Allgemeinheit getragen werden, darf der Entscheid für die Therapien wohl individuell gefällt werden. Wenn bei fehlendem belegbarem Nutzen der Anspruch erhoben wird, Krankheiten zu behandeln (Divertikelleiden, chronische Entzündungen), ist dies problematischer.
5 Schlaeppi M: Entwicklung von integrativer Medizin bedeutet auch Verantwortung für Qualität. Schweiz Z Ganzheitsmed 2016;28: 317.

6 Biedermann L, Rogler G, Zeitz L: «Mikrobiota-Tuning» en vogue: Von der Ernährung über Probiotika bis zur fäkalen MikrobiotaTransplantation. Schweiz Z Ganzheitsmed 2017;29:DOI: 10.1159/000475586.

7 Acosta RD, Cash BD: Clinical effects of colonic cleansing for general health promotion: a systematic review. Am J Gastroenterol 2009; 104:2830-2836.
8 Jalanka J, Salonen A, Salojärvi J, et al: Effects of bowel cleansing on the intestinal microbiota. Gut 2015;64:1562-1568.

9 Drago L, Toscano M, De Grandi R, et al: Persisting changes of intestinal microbiota after bowel lavage and colonoscopy. Eur J Gastroenterol Hepatol 2016;28:532-537.

10 Frei P, Rogler G: Rektale Therapie bei chronisch entzündlichen Darmerkrankungen. Broschüre. Dr. Falk Pharma GmbH, 2016.

11 Ernst E: Colonic irrigation: therapeutic claims by professional organisations, a review. Int J Clin Pract 2010;64:429-431. 